\title{
Automated Emergency Vehicle Control Strategy Based on Automated Driving Controls
}

\author{
Jaehyun (Jason) So $\mathbb{D},{ }^{1}$ Jiwon Kang, ${ }^{2}$ Sangmin Park $\mathbb{D},{ }^{3}$ Inseon Park $\mathbb{D},{ }^{4}$ \\ and Jongdeok Lee $\mathbb{D}^{5}$ \\ ${ }^{1}$ The Korea Transport Institute, Room 215, 370 Sicheong-daero, Sejong, Republic of Korea \\ ${ }^{2}$ The Korea Transport Institute, Room G104, 370 Sicheong-daero, Sejong, Republic of Korea \\ ${ }^{3}$ Smart Transportation Engineering Lab, Ajou University, 206, World Cup-ro, Yeongtong-gu, Suwon-si, \\ Gyeonggi-do, Republic of Korea \\ ${ }^{4}$ Dream ENG Co., 59, Oncheon-ro, Yuseong-gu, Daejeon, Republic of Korea \\ ${ }^{5}$ The Korea Transport Institute, Room 425, 370 Sicheong-daero, Sejong, Republic of Korea
}

Correspondence should be addressed to Jaehyun (Jason) So; jso@koti.re.kr

Received 31 October 2019; Accepted 26 December 2019; Published 1 February 2020

Guest Editor: Inhi Kim

Copyright ( 2020 Jaehyun (Jason) So et al. This is an open access article distributed under the Creative Commons Attribution License, which permits unrestricted use, distribution, and reproduction in any medium, provided the original work is properly cited.

\begin{abstract}
This study proposes an integrated driving control strategy by taking advantage of the automated driving technology at the individual vehicle level and the traffic signal preemption strategy at the traffic infrastructure level. This aims to facilitate an automated drivingbased emergency vehicle control and ultimately to achieve efficient and safe control of emergency vehicles. To this end, this study developed the integrated emergency vehicle control logic, implemented the logic in the microscopic traffic simulation environment using the simulation software's application programming interface capability, and evaluated the impacts of the proposed emergency vehicle control logic in the aspects of mobility and safety with different driving aggressiveness and preemption initiation settings. The study's results show that the proposed emergency vehicle control logic achieved benefits on mobility and safety and the benefits of emergency vehicle control strategy can be maximized when the signal preemption and the automated driving control operate in collaboration. Therefore, the proposed integrated approach of automated driving controls and signal preemption will be a great reference for enhancing automated driving technologies supporting a safe and fast mobility solution.
\end{abstract}

\section{Introduction}

Automated driving technologies have been spotlighted in recent years. Safety systems and applications are the core of the automated driving technology because safety is a critical value when a new transportation mode is deployed in reality. This emphasis on safety could reduce mobility on roadways due to frequent decelerations against projected dangers, indicating that there is a tradeoff between safety and mobility. To reduce this tradeoff and enhance the performance of automated driving technology in both safety and mobility, this study adopts an emergency vehicle (hereafter "EMV") as a representative traffic application that needs to achieve both mobility and safety at the same time. The emergency vehicles such as ambulances and police vehicles should reach the designated destinations fast and safe.

By focusing on the importance of EMVs, some previous studies developed traffic signal preemption strategies by manipulating traffic signal parameters in order to provide an exclusive passage to EMVs [1-4]. The recent studies took advantage of the wireless communications technology such as vehicle-to-vehicle (V2V) and vehicle-to-infrastructure (V2I) communications (hereinafter "V2X") in order to transmit the EMV's location information to traffic signal controllers in advance to reach the intersections. Based on these examples, it is expected that support from road infrastructure such as V2X communications and traffic signal controls can facilitate the operation of EMVs [5-8]. 
To sum up, this study takes advantage of the automated driving technology at the individual vehicle level and the traffic signal preemption strategy at the traffic infrastructure level in order to develop the automated EMV control strategy and ultimately to facilitate mobile and safe control of automated vehicles. Hence, this study develops the integrated EMV control algorithm and investigates the impacts of the EMV algorithm on mobility and safety using multiple driving aggressiveness scenarios. Furthermore, this study takes the optimum driving parameters set concerning mobility and safety performance measures into consideration.

\section{Literature Review}

2.1. Automated Vehicle Control Models. Vehicle automation has seen unprecedented development in the recent years. Levinson et al. [9] have shown the system structure and control algorithms of an automated vehicle. In order to simulate it in a microscopic traffic simulation context, the simulated vehicle has to imitate the automated vehicle's longitudinal and lateral movement behavior. In terms of longitudinal control or car-following behavior, unlike a human driver, who has a control variance, often modeled by Wiedemann74 model [10] or Gipps model [11], automated vehicles have almost zero latency and control variance. Kesting et al. [12] have presented the Enhanced Intelligent Driver Model (EIDM), which represents the car-following behavior of a vehicle with adaptive cruise control (ACC). Based on Kesting et al.'s EIDM, the following vehicle adjusts its acceleration based on the leading vehicle's speed, acceleration, distance, and its own speed, maintaining an acceptable following distance.

In terms of lateral control or lane change behavior, there are less intensive researches on this topic. Within traffic simulations, lane changes are modeled using a rule-based decision process [13], which looks into the necessity, possibility, and benefit of a proposed lane change action. Kesting et al. [14] proposed another gap based lane change model, which aims at minimizing breaking distance. This model, though, assumes that other vehicles in the vicinity follow the Intelligent Driver Model [15]; thus it is unsuitable for lane change decision-making, where nearby vehicles adopt a carfollowing behavior based on the Wiedemann model. Naranjo et al. [16] have presented a lane change mechanism based on fuzzy logic; it provides a smooth transition from one lane to another, whereas it assumes a relatively static nearby vehicle movement. Recent studies have moved to using neural networks to address this issue; Ulbrich and Maurer [17] propose a probabilistic decision network for the tactical lane change decision process. Two independent signal processing networks have been proposed to assess the nearby vehicles in different regions of interests (ROI). The output is the probability for whether a lane change is possible and the probability for whether a lane change is beneficial.

2.2. Traffic Signal Preemption. The first electric traffic signal was installed in 1914 in Cleveland and was equipped with a manual switch for firemen, which led to red signals in all approaches to facilitate the passage of the fire engine [18]. Beyond this manual signal preemption operation, three main technologies for automatic detection of emergency vehicles at signalized intersections have evolved and are used in practice. The first technology in use is based on the siren sound that is detected with directional microphones in order to determine the direction from which the emergency vehicle is approaching [3]. The second technology is based on emission of light or infrared strobes by the emergency vehicles that are detected by dedicated detectors located at the signal head. A third technology is based on radio transmission, which requires a separate technology for the positioning of the vehicle such as GPS or infrared beacons.

With Dedicated Short Range Communication (DSRC), a new technology is arising, which takes the radio based communication to a new level with higher bandwidths allowing an exchange of information with increasing volume. The evolving standards $[19,20]$ foresee data elements for Signal Request Messages (SRM) as well as a Signal Status Message (SSM) acknowledging the request. Furthermore, Emergency Vehicle Alerts (EVA) can be broadcasted by emergency vehicles to other road users in the vicinity in order to raise their awareness.

These novel functionalities are investigated in several research projects with different signal control algorithms including ImFlow [21] and Multi-Modal Intelligent Transportation Signal System (MMITSS) with its application Emergency Vehicle Priority (PRE-EMPT) [22].

\section{Methodology}

3.1. Study Assumption. This study develops the EMV control strategy by integrating automated driving technology and the existing traffic signal preemption strategy. While the EMV control strategy proposed in this study adopts state-ofthe-art technologies, some technical assumptions are made in this study as follows:

(i) The EMV is equipped with a GPS device, capable of positioning the vehicle's location

(ii) The EMV is equipped with an on-board unit (OBU), capable of V2X communication

(iii) The EMV is equipped with vehicle sensors, capable of detecting adjacent vehicles

(iv) Traffic signal controllers in this road network are equipped with roadside units (RSU), capable of receiving the EMV's wireless transmission

In addition to the assumptions on technical requirements, further assumptions were made in terms of the EMV's driving maneuver as follows: the EMV has a desired speed of $100 \mathrm{~km} /$ $\mathrm{h}$; the EMV drives within a normal driving concept, and no special right-of-way (ROW) is allowed to the EMV. For example, the EMV follows traffic signals, drives only within a lane, and is not allowed to drive on a contraflow lane; and adjacent vehicles respond to the EMV in a normal way based on the VISSIM's off-the-shelf car-following behaviors and do not take evasive maneuvers (e.g., stopping and evasive road 
departure) against the EMV. This is because this study focuses on the utilization of automated driving controls for the purposes of both mobility and safety, rather than the specific driving maneuvers of EMV. In other words, this study removes other external impacts such as the EMV's special ROW and the normal vehicles' evasive maneuvers and only investigated the EMV's maneuver scenarios by different setting of automated driving control parameters.

3.2. Automated Driving Controls. An Enhanced Intelligent Driver Model (EIDM) is used for the vehicle's longitudinal control; for the vehicle's lateral control, a probabilistic lane change decision process is implemented. A set of aggressiveness levels are defined in order to evaluate the mobility and safety impact of driving strategies according to these levels.

3.2.1. Longitudinal Behavior Model. In order to model the car-following behavior of an automated vehicle, one needs to

be aware of the significant differences from a human driver. Automated vehicles, compared to cars with human drivers, can be assumed to have zero reaction time, zero control variance, and no loss of attention.

The EIDM [17] is a time-continuous car-following model representing the ACC driving behavior; it also serves as the basis of an ACC implementation of real vehicles. The model extends the Intelligent Driver Model (IDM) presented by Treiber et al. [15] with a constant-acceleration heuristic (CAH). This avoids the formerly observed, sometimes unrealistic, behaviors in noncritical braking situations, for example, when a car changes lanes in front of another vehicle, causing the gap to be less than desired. The EIDM has inherited IDM's intuitive behavioral parameters: desired velocity, acceleration, comfortable deceleration, and desired minimum time headway.

The CAH and EIDM are given in the following equations:

$$
\begin{aligned}
a_{\mathrm{CAH}}\left(s, v, v_{l}, a_{l}\right)= \begin{cases}\frac{v^{2} \widetilde{a}_{l}}{v_{l}^{2}-2 s \tilde{a}_{l}+\varepsilon}, & \text { if } v_{l}\left(v-v_{l}\right) \leq-2 s \tilde{a}_{l}, \\
\tilde{a}_{l}-\frac{\left(v-v_{l}\right)^{2} \Theta\left(v-v_{l}\right)}{2 s}, & \text { otherwise, }\end{cases} \\
a_{\mathrm{EIDM}}= \begin{cases}a_{\mathrm{IDM}}, & \text { if } a_{\mathrm{IDM}} \geq a_{\mathrm{CAH}}, \\
(1-c) a_{\mathrm{IDM}}+c\left[a_{\mathrm{CAH}}+b \tanh \frac{\left.a_{\mathrm{IDM}}-a_{\mathrm{CAH}}\right],}{b}\right. & \text { otherwise. }\end{cases}
\end{aligned}
$$

The CAH determines the maximum acceleration $a_{\mathrm{CAH}}$ leading to no crashes. "The condition $v_{l}\left(v-v_{l}\right) \leq-2 \mathrm{~s} v_{l}(v-$ $\left.v_{l}\right) \leq-2 s \tilde{a}_{l}$ is true if the vehicles have stopped at the time the minimum gap $s=0$ is reached" [12]. The Heaviside step function $\Theta(x)$ is used for eliminating negative approaching rates. The term $\tilde{a}$ is the minimum value of $a$ and $a_{l}$, which are the accelerations of the subject vehicle and the leading vehicle, respectively, and $\varepsilon$ is an extremely small number to prevent the denominator from being equal to zero. The acceleration of the ACC vehicle is expressed by $a_{\text {EIDM }}$. This again has two cases; only if the acceleration computed by the IDM is unrealistic is the second term in equation (2) used. The parameter $c$ of this formula is a "coolness factor," which determines the weights placed on CAH and IDM.

Finally, the parameters are set as follows:

(i) Desired speed $\left(v_{0}\right): 100 \mathrm{~km} / \mathrm{h}$

(ii) Free acceleration exponent $(\delta): 3$

(iii) Desired time gap ( $T): 0.5 \mathrm{~s}$

(iv) Minimum standing distance $\left(s_{0}\right): 2.0 \mathrm{~m}$

(v) Maximum acceleration $(a): 4.2 \mathrm{~m} / \mathrm{s}^{2}$

(vi) Desired deceleration $(b): 4.0 \mathrm{~m} / \mathrm{s}^{2}$

(vii) Coolness factor (c): 0.99
In order to demonstrate the difference between the EIDM and the Wiedemann model, which is the default carfollowing model in VISSIM, a testing scenario is created to reveal the responses given by the two models to a leading vehicle. The leading vehicle in this case uses the Wiedemann model and is placed 100 meters ahead of the subject vehicle to test the behavior in car following. The leading vehicle would undergo several changes of desired speeds. The reactions of the subject vehicle using different models are illustrated in Figure 1. The EIDM vehicle indicates higher maximum speeds and smoother accelerations/decelerations.

3.2.2. Lateral Behavior Model. As in Ulrich and Maurer [17], two signal processing networks are used to determine if a lane change is possible and beneficial with probabilistic outputs. These two signal processing networks were used as part of a neural network model; however, in this paper, the signal processing networks are implemented as the solo criteria for lane changing decisions with predefined thresholds.

The signal processing networks comprise two parts: a network for "lane change possible" decision process and a network for "lane change benefit" decision process. The lane 


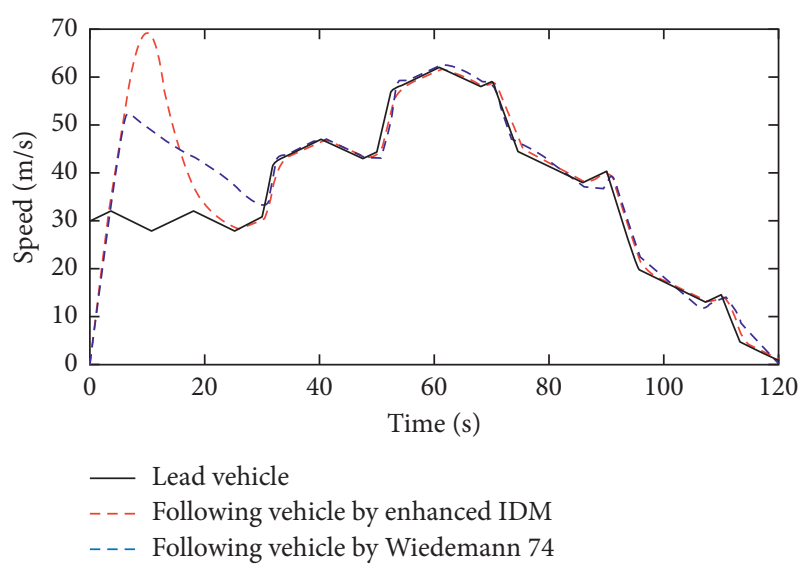

Figure 1: Comparison of car-following models.

change possibility is based on the dynamic vehicle in front of the subject vehicle on its own lane, the vehicle in front of the subject vehicle on its neighbor lane, and the vehicle behind the subject vehicle on its neighbor lane. The lane change benefit considers the direct front vehicle on the same lane and the front vehicle on the neighbor lane. If any front vehicle does not exist, then the benefit probability is set to zero directly. Note that only a 2-lane street is considered in the case study.

A threshold for each signal network is given based on aggressiveness levels, which dictates when a lane change action should be executed. This signal network considers relative distances, relative velocities, and time to collision with nearby vehicles around the subject vehicle.

The signal processing network for calculating an aggregated probability measurement to determine whether a lane change is possible is a mathematical calculation, aggregating each situation in the regions of interest (ROI). Each situation expresses its own possibility of performing a lane change on a scale from zero to one. The final result indicates the aggregated possibility. If no object exists in the ROI, the likeliness that a lane change is possible is directly set to 1 . If an object does exist, then a series of cumulative Gaussian distributions translate the numeric values of the object attributes into a specific number value in the scale of zero and one, based on $\mu$ and $\sigma$ given in each distribution. Object attributes include speed, distance, time gap $\left(t=\right.$ distance $\left./ v_{\text {ego }}\right)$, and time to collision $($ TTC $=$ distance $/$ $\left.\left(v_{\text {obj }}-v_{\text {ego }}\right)\right)$. After calculating all the cumulative distribution functions, the intermediate results are aggregated into one measurement to represent a particular ROI, and finally a minimal value is selected from all ROI as the final probability for "lane change possible."

3.2.3. Driving Aggressiveness Settings. Driving aggressiveness can be defined with different parameter sets for the carfollowing and lane change models. The strategy in defining these driving aggressiveness levels was to make the EMV proactively take lane changes under maximum allowable maneuvering capacity. Therefore, the car-following parameters (i.e., desired time gap $\lambda_{T}$, maximum acceleration rate $\lambda_{a}$, and desired deceleration rate $\lambda_{b}$ ) were set at maximum as follows in order to allow a full driving capacity of the EMV:

(i) Desired time gap $\left(\lambda_{T}\right): 0.5$ seconds

(ii) Maximum acceleration rate $\left(\lambda_{a}\right): 4.2 \mathrm{~m} / \mathrm{s}^{2}$

(iii) Desired deceleration rate $\left(\lambda_{b}\right): 4.0 \mathrm{~m} / \mathrm{s}^{2}$

The aggressiveness levels were thus defined with different lane change criteria (i.e., lane change possibility and benefit probabilities), and the lane change possibility and benefit probabilities were assumed to be realistic in the range of $20 \%$ and $80 \%$. The rationale of this boundary is based on the following assumptions: lane changes happen too frequently if the lane change criteria are set to happen when both the possibility and benefit probabilities are lower than $20 \%$, and, in contrast, no lane changes would occur if the lane change criteria are set to happen when both the possibility and benefit probabilities are higher than $80 \%$. Therefore, the driving aggressiveness levels were set with 16 different combinations of four different lane change possibility probability criteria $(20 \%, 40 \%, 60 \%$, and $80 \%)$ and four different lane change benefit possibility criteria $(20 \%, 40 \%$, $60 \%$, and $80 \%$ ). Figure 2 shows the selection concept of driving aggressiveness levels. The " $20 \%$ of possibility probability and $20 \%$ of benefit probability" criteria scenario represents the most aggressive driving maneuver, while lane changes happen in the most conservative manner in the " $80 \%$ of possibility probability and $80 \%$ of benefit probability” criteria scenario.

3.3. Signal Preemption. An emergency vehicle signal preemption (EVSP) strategy is designed to provide a signal priority to EMVs approaching a signalized intersection. The general logic of EVSP operates with the following processes: a request for preferential signal is transmitted to a traffic signal controller located at a specific intersection, when an EMV approaches the signalized intersection; once the signal controller receives the signal from the EMV including its location, the controller initiates the preset EVSP program; the EVSP program estimates the appropriate timing for green indication by estimating the queue discharge time and the arrival time of the EMV based on the EMV's location information; and the EVSP program provides green signal to the EMV's approach at the estimated timing in order for the EMV to pass through the intersection without delay.

This study adopted a coordinated dynamic traffic signal preemption strategy taking advantage of Intelligent Transportation Systems (ITS) technologies [2]. Basic criteria of EVSP are to make EMVs pass through a signalized intersection without delay and to minimize the side effect of EVSP on the other traffic. To meet these criteria, the green signal should be indicated at the effective timing after the queued vehicles and the moving vehicles are discharged. The EVSP in this study utilizes the dynamic notification time concept as shown in Figure 3 by estimating the remaining time for the EMV to arrive at the intersection.

To this end, the notification time is estimated based on switchover time, queue discharge time, and safety time 

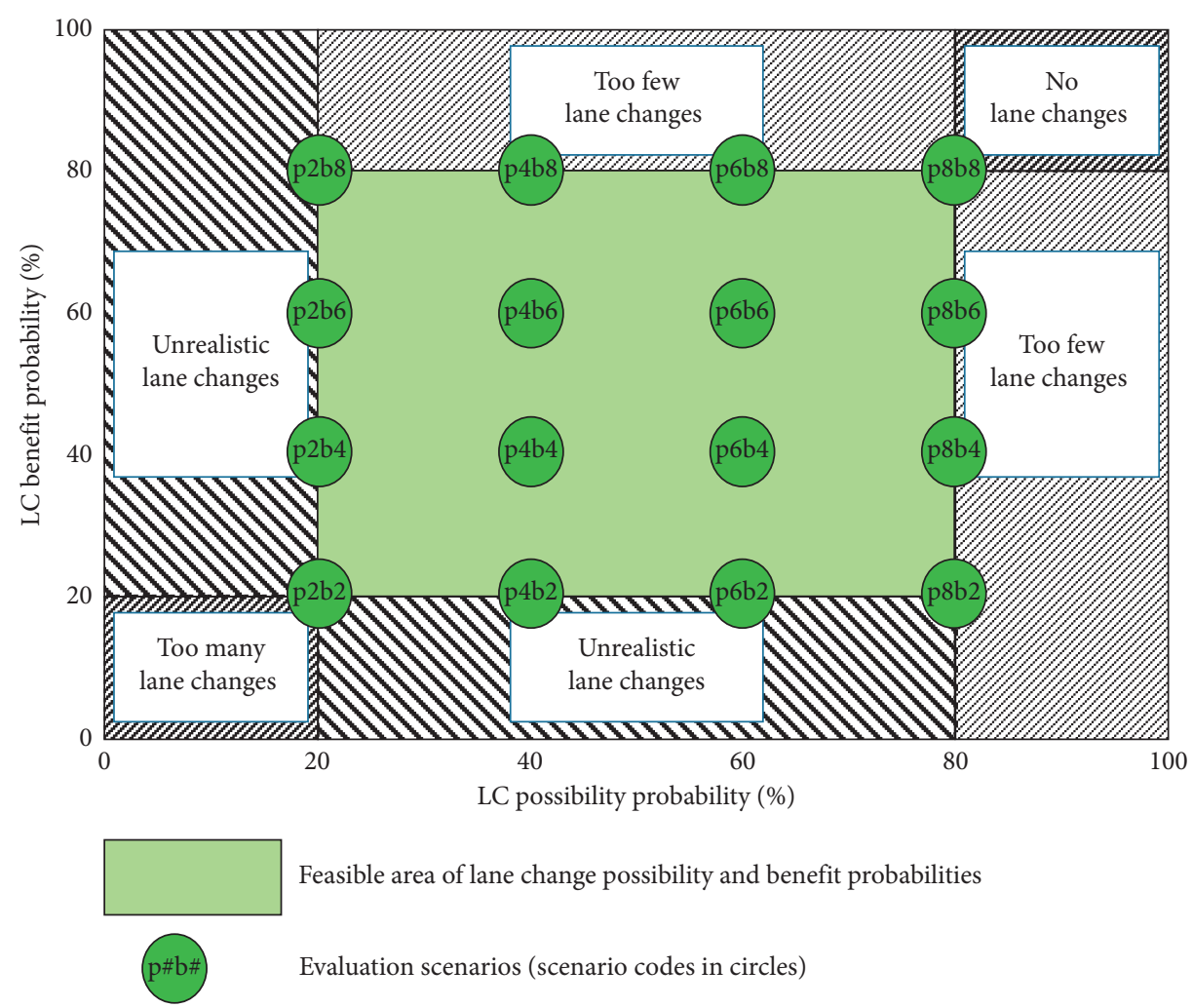

Figure 2: Descriptive concept of the evaluation scenarios.

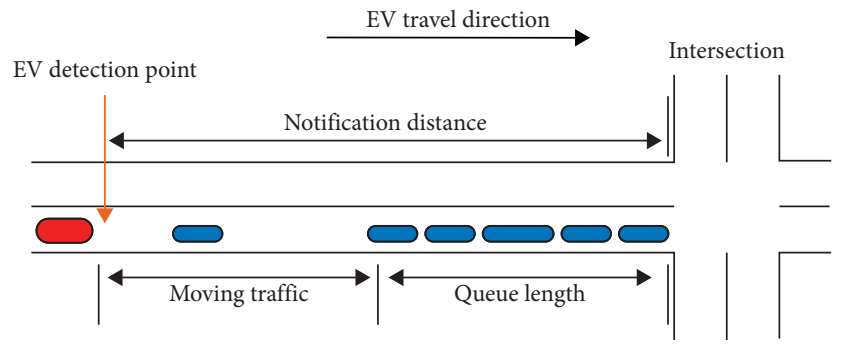

Figure 3: Dynamic notification distance based on queue length.

interval, as shown in equation (3). The switchover time is the sum of inter-green time and minimum green time; the discharge time is the amount of time required for queued traffic to be discharged; and the safety time interval is a time interval between the last queued vehicle and the EMV assuring a fluent passage and safety of the EMV. The safety time interval is assumed to be 3 seconds in this study.

Notification time $=$ switchover time + discharge time

$$
+ \text { safety time interval. }
$$

\subsection{Simulation Implementation}

3.4.1. Traffic Simulation Model. To implement and evaluate the EMV control strategy proposed in this study, a microscopic traffic simulation approach is applied. VISSIM 10.0 [23] was selected due to its extensive capability of modeling traffic situations and implementing automated vehicle controls. VISSIM provides a component object model (COM) interface, which facilitates traffic controls based on the user's specific logic using programming languages. In addition, VISSIM also provides a driver model DLL file enabling users to interrupt off-the-shelf driver behavior logics in VISSIM and control vehicles as programmed and a vehicle actuated programming (VAP) module to manipulate traffic signal timings based on the detectors' information and the user's logic. Regarding the fact that the EMV control strategy in this study requires not only modeling of base traffic situations but also signal preemption and automated driving controls, VISSIM is an appropriate simulation software for the experiments in this study.

3.4.2. Network Modeling. The EMV control strategy proposed in this study was implemented and tested in a microscopic traffic simulation network representing the Frankfurter Ring road section, located in the city of Munich, Germany. The selected road section has three signalized intersections where Schleissheimer Str., Knorrstr., and Ingolstaedter Str. (from left) intersect. The stretch of this simulation network is $4.18 \mathrm{~km}$ from the west-most point to the east-most point, and the network was built using VISSIM as shown in Figure 4.

Data collection was made by Automatic Number Plate Recognition (ANPR) and manual measurements in order to build and calibrate a simulation network. The measurements took place from 8 AM to 9 AM on October 27, 2014, and each ANPR camera was located on the Frankfurter Ring road, while the other side roads were manually observed. In 


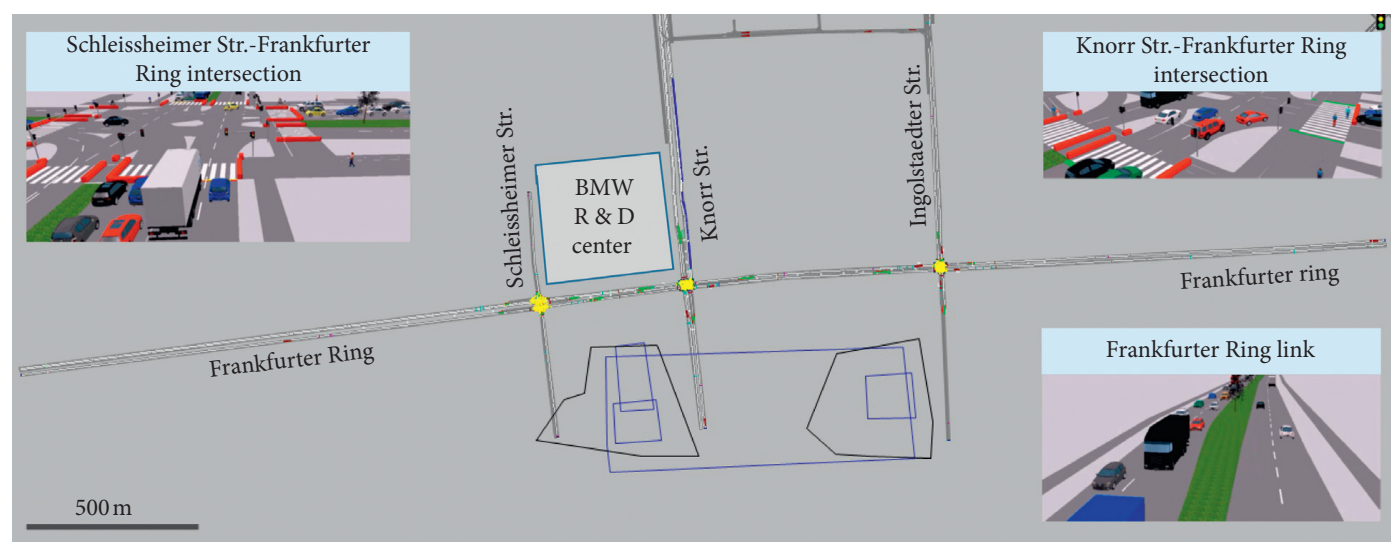

FIgURE 4: Frankfurter Ring VISSIM network.

addition, road geometry and traffic signal timing plan data were provided by the city of Munich. Finally, traffic volume, speed, travel time, and signal timing parameter data were collected, and these were used to build and calibrate the VISSIM network [24].

The model was calibrated and validated by adjusting carfollowing parameters in order to assure the model's reliability representing actual traffic situations in reality. For the calibrated parameters, standstill distance (CC0), headway time (CC1), following variation (CC2), negative following threshold (CC4), and positive following threshold (CC5) are used and adjusted based on turning counts, queue lengths, and travel times collected in the field [25]. The final model was satisfied with the calibration stopping criteria, since $R^{2}$ values were higher than 0.90 in the all measurements (i.e., turning volumes, queue lengths, and travel times) [24]. In addition, the model was fine-tuned using reduced speed areas and desired speed distributions in VISSIM in order to enhance the model's reliability.

3.4.3. Simulation Settings. Twelve simulation runs were made to capture variability in the simulation runs. The number of simulation runs was determined based on the computed sample size, and twelve simulation runs were statistically sufficient to cover the variability of this simulation at a 95\% confidence level [26]. The entire simulation period was set to 1,800 seconds, while first 1,200 seconds of warm-up time were used to fill up the network with vehicles and the remaining 600 seconds of simulation period were used to evaluate the impact of the EMV.

3.4.4. Evaluation Settings. A total of 18 scenarios were set to assess the impact of the EMV control proposed in this study as shown in Table 1. Scenario 1 (base) does not implement any special treatment for EMVs, but the EMV is set to drive at $100 \mathrm{~km} / \mathrm{h}$ by desire. Scenario 2 reflects a treatment at the infrastructure level and utilizes signal preemption for the EMV to pass through intersections without delay.

This scenario represents a conventional signal preemption strategy, which is the state-of-the-art treatment for EMVs in the past years. The other 16 scenarios utilize both signal preemption and automated controls and are set with different driving aggressiveness levels based on the lane change possibility and benefit probabilities, as defined in the previous section.

3.4.5. Driving Aggressiveness Settings. Scenario 3 reflects the most aggressive driving maneuver, which triggers lane changes if both the estimated lane change possibility and benefit probabilities are more than $20 \%$. Scenario 18 represents the most conservative driving maneuver, which triggers lane changes only if the two probabilities are more than $80 \%$. In addition, the EMV entered into the network at a different state of the traffic signal by increasing 10 seconds in each scenario in order to consider the influence of interruption timing in the signal cycle length. Since the intersections in the test-bed network operate with 90 seconds of cycle length, nine times of simulation run were implemented for each evaluation scenario.

3.4.6. Measures of Effectiveness. The impact of the EMV control proposed in this study was evaluated in terms of mobility and safety. The mobility impact was evaluated at both the network-wide level and the individual EMV level; the network-wide average delay was measured for the network-wide impact assessment; and travel time, average speed, and average delay were measured for the individual EMV. For the safety impact assessment, traffic conflicts, a probability of crashes, were estimated using vehicle trajectories extracted from VISSIM. A surrogate safety assessment model (SSAM) [27] is used to compute surrogate safety measures such as time-to-collision (TTC) and postencroachment time (PET) and estimate traffic conflicts. A TTC value of less than or equal to 1.5 seconds and a PET value of less than or equal to 5.0 seconds were used as the thresholds for identifying traffic conflicts.

\section{Analysis and Evaluation}

4.1. Driving Maneuver. Average 0.5 lane change occurred in the base and 4 lane changes occurred in the signal preemption scenario. This shows that signal preemption was effective by providing green signal to EMV, while less than 
TABLE 1: Evaluation scenarios.

\begin{tabular}{|c|c|c|c|c|c|}
\hline \multirow{2}{*}{\multicolumn{2}{|c|}{ Scenarios }} & \multirow[b]{2}{*}{ Scenario code } & \multirow[b]{2}{*}{ Signal preemption } & \multicolumn{2}{|c|}{ Automated driving } \\
\hline & & & & $\begin{array}{l}\text { LC possibility } \\
\text { criteria }(\%)\end{array}$ & $\begin{array}{l}\text { LC benefit } \\
\text { criteria (\%) }\end{array}$ \\
\hline \multicolumn{2}{|c|}{ Scenario 1 (base) } & Base & Not applied & - & - \\
\hline \multicolumn{2}{|c|}{ Scenario 2 (signal preemption only) } & SP & Applied & - & - \\
\hline \multirow{16}{*}{$\begin{array}{l}\text { Signal preemption }+ \\
\text { automated control }\end{array}$} & Scenario 3 & $\mathrm{p} 2 \mathrm{~b} 2$ & Applied & 20 & 20 \\
\hline & Scenario 4 & $\mathrm{p} 2 \mathrm{~b} 4$ & Applied & 20 & 40 \\
\hline & Scenario 5 & p2b6 & Applied & 20 & 60 \\
\hline & Scenario 6 & p2b8 & Applied & 20 & 80 \\
\hline & Scenario 7 & $\mathrm{p} 4 \mathrm{~b} 2$ & Applied & 40 & 20 \\
\hline & Scenario 8 & $\mathrm{p} 4 \mathrm{~b} 4$ & Applied & 40 & 40 \\
\hline & Scenario 9 & p4b6 & Applied & 40 & 60 \\
\hline & Scenario 10 & $\mathrm{p} 4 \mathrm{~b} 8$ & Applied & 40 & 80 \\
\hline & Scenario 11 & p6b2 & Applied & 60 & 20 \\
\hline & Scenario 12 & p6b4 & Applied & 60 & 40 \\
\hline & Scenario 13 & p6b6 & Applied & 60 & 60 \\
\hline & Scenario 14 & p6b8 & Applied & 60 & 80 \\
\hline & Scenario 15 & $\mathrm{p} 8 \mathrm{~b} 2$ & Applied & 80 & 20 \\
\hline & Scenario 16 & $\mathrm{p} 8 \mathrm{~b} 4$ & Applied & 80 & 40 \\
\hline & Scenario 17 & p8b6 & Applied & 80 & 60 \\
\hline & Scenario 18 & $\mathrm{p} 8 \mathrm{~b} 8$ & Applied & 80 & 80 \\
\hline
\end{tabular}

one lane change occurs if there is no interruption on traffic signal and driving behavior parameters (i.e., base scenario). For the automated control scenarios, 7 lane changes occurred when the lane change criteria were $20 \%$ of possibility and $20 \%$ of benefit; only 1 lane change occurred when either the lane change possibility criteria or the lane change benefit criteria were $80 \%$; and the other automated control scenarios were between these two cases in the range from 1 time to 7 times, as shown in Figure 5(a). The results seem reasonable because lane changes would hardly occur if the lane change criteria are high (i.e., the most conservative behavior) and frequently occur if the lane change criteria are low (i.e., the most aggressive behavior).

The average speed of the EMV (Figure 5(b)) was the lowest in the base scenario because the EMV had to frequently stop due to red signals (no preemption). Average speed in the preemption scenario was higher than that of the base scenario because of the effective passage by the benefit of signal preemption. In the automated-control-integrated scenarios (i.e., integration of the automated control and the signal preemption), most scenarios showed higher average speed compared to the signal-preemption-only scenario, while the average speed in the conservative driving aggressiveness scenarios, in which the lane change criteria are higher than $60 \%$ in either possibility or benefit, was similar to the signalpreemption-only scenario in the range of $5 \%$. This indicates that EMVs can drive faster with reasonable lane changes (i.e., neither too many nor too few lane changes).

The average acceleration rates (Figure 5(c)) were similar in all scenarios, but the ranges of acceleration rates in the base and signal preemption scenarios were a bit smaller than those in the other automated control scenarios. This is because the maximum acceleration rate and the desired deceleration rate were set at maximum in the automated control scenarios in order to allow a full driving capacity of EMV.
4.2. Mobility Impact. Based on delay and travel time measurements (Figures 6(a) and 6(b)), the EMV was faster than the other normal vehicles (i.e., black dots mean average delay of normal vehicles) in the range from $66.7 \%$ to $87.7 \%$. Particularly, the "p2b4" scenario was the best and had 35.2\% less delay compared to normal vehicles; the "p2b2," "p2b6," "p2b8," and "p4b2" scenarios followed (less delay in the range from $35.3 \%$ to $40.8 \%$ compared to normal vehicles); and the EMVs of the automated control scenarios that were set with $80 \%$ of lane change possibility or benefit criteria showed a better performance than the base scenario in terms of delay and travel time, but the performance was not significant compared to the other automated control scenarios. This is because EMV was relatively steady in the conservative scenarios and travelled with less lane changes in response to sluggish vehicles in forward.

4.3. Safety Impact. Traffic conflicts varied in the range from 1,184 to 1,438 in the network as shown in Figure 6(c), and the impact of automated vehicle control in the entire network appeared to be insignificant in terms of safety. Meanwhile, the automated vehicle control decreased traffic conflicts, since the numbers of conflicts involving EMV ranged from 4.4 to 7.6 in the automated control scenarios, while 8.9 conflicts were found in the base scenario.

4.4. Discussion. The findings based on the mobility and safety impact assessment results are discussed as follows:

(i) Increasing desired speed is effective to improve mobility of EMV by approximately $10 \%$ (i.e., base); signal preemption is effective for the mobility of EMV; and the integration of a signal preemption (treatment at road infrastructure) and an automated vehicle control (treatment at individual vehicle) 


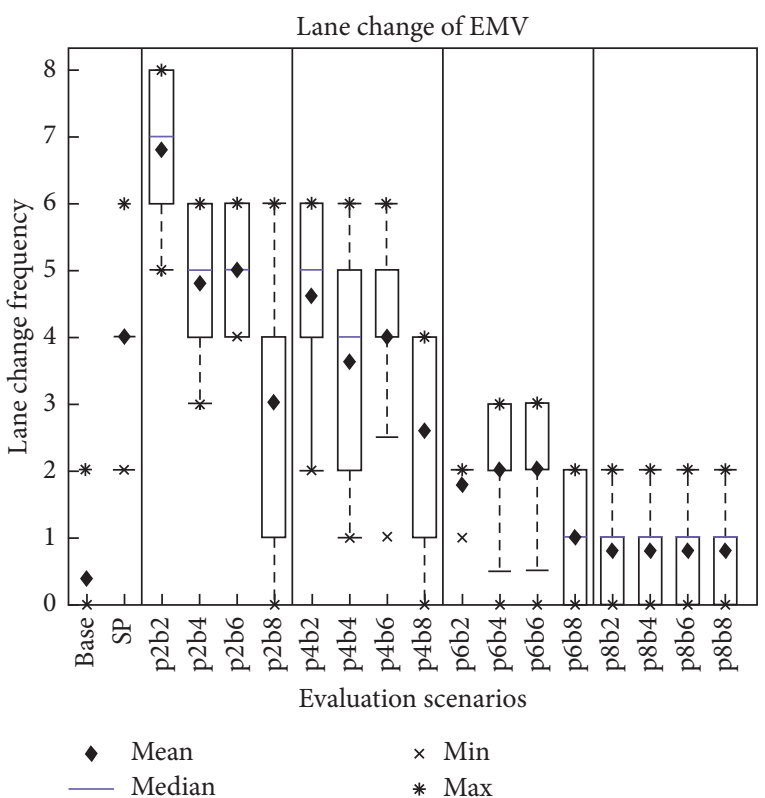

(a)

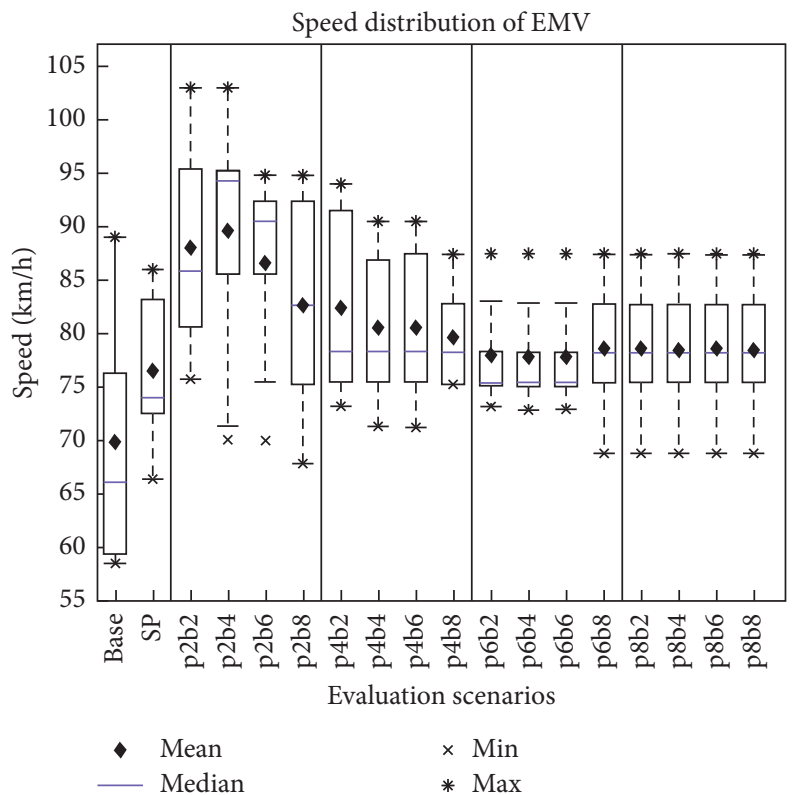

(b)

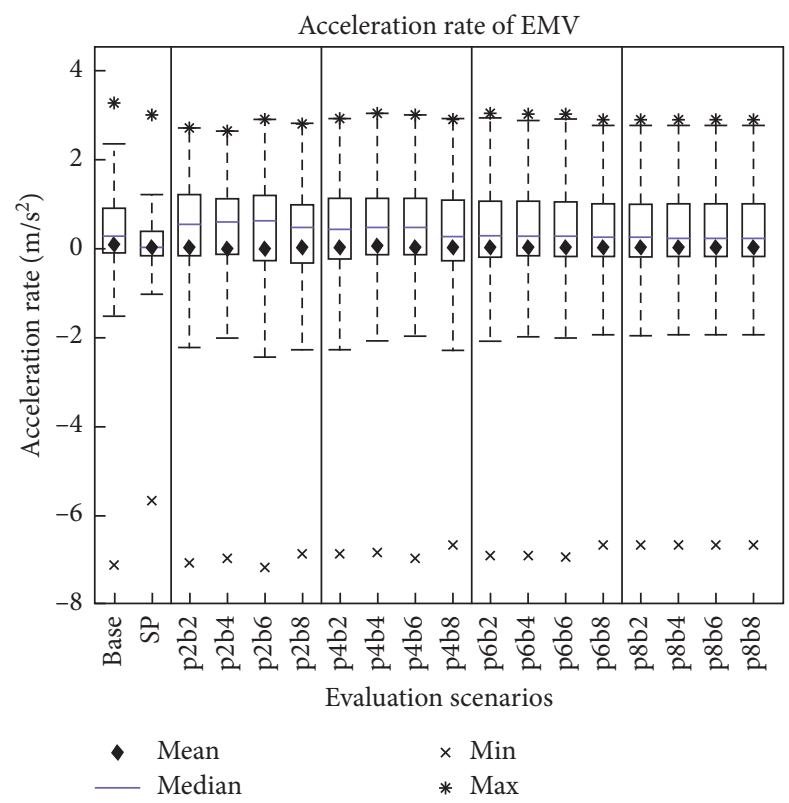

(c)

FIGURE 5: Driving maneuvers. (a) Lane change frequency. (b) Speed. (c) Acceleration rate $\left(\mathrm{m} / \mathrm{s}^{2}\right)$.

significantly enhances the mobility of EMV by decreasing delay.

(ii) An integrated approach of an automated vehicle control and a signal preemption is beneficial for EMVs to reduce conflicts involving adjacent vehicles, while it does not provide significant side impact in the entire network.

(iii) A signal preemption is beneficial for EMV in terms of mobility and safety, but the impact can be maximized in integration with an automated vehicle control at the individual vehicle level. (iv) The impact of automated vehicle control varies by different driving aggressiveness levels in terms of mobility and safety. For example, the most aggressive setting resulting in many lane changes performs well in terms of the mobility measures such as travel time and delay, while the most conservative setting resulting in few lane changes performs well in terms of safety. Therefore, the impact of automated vehicle control can be maximized with reasonable level of the driving aggressiveness setting (i.e., lane change criteria in this study) in consideration of both mobility and safety. 


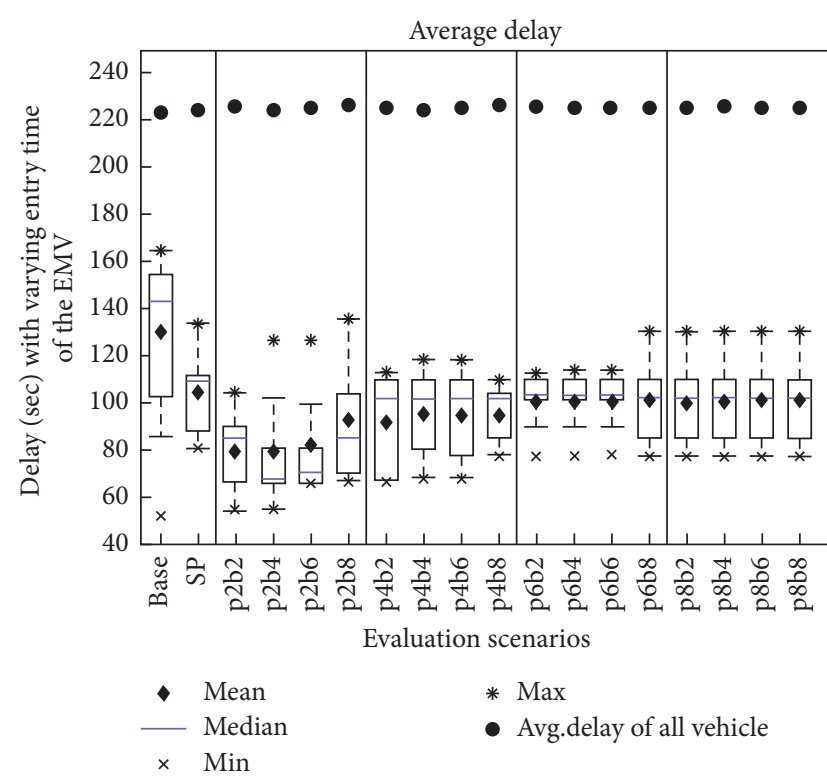

(a)

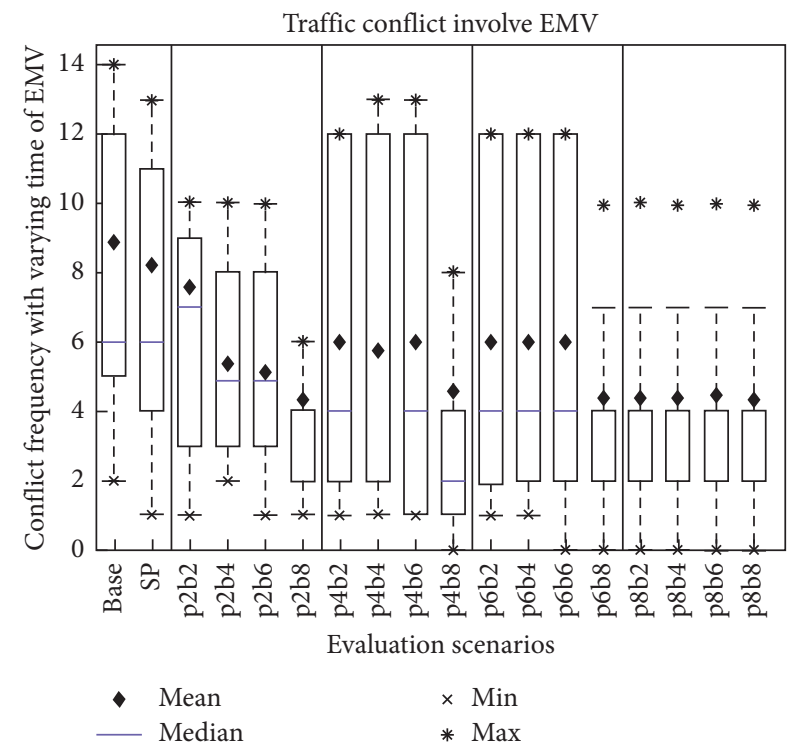

(c)

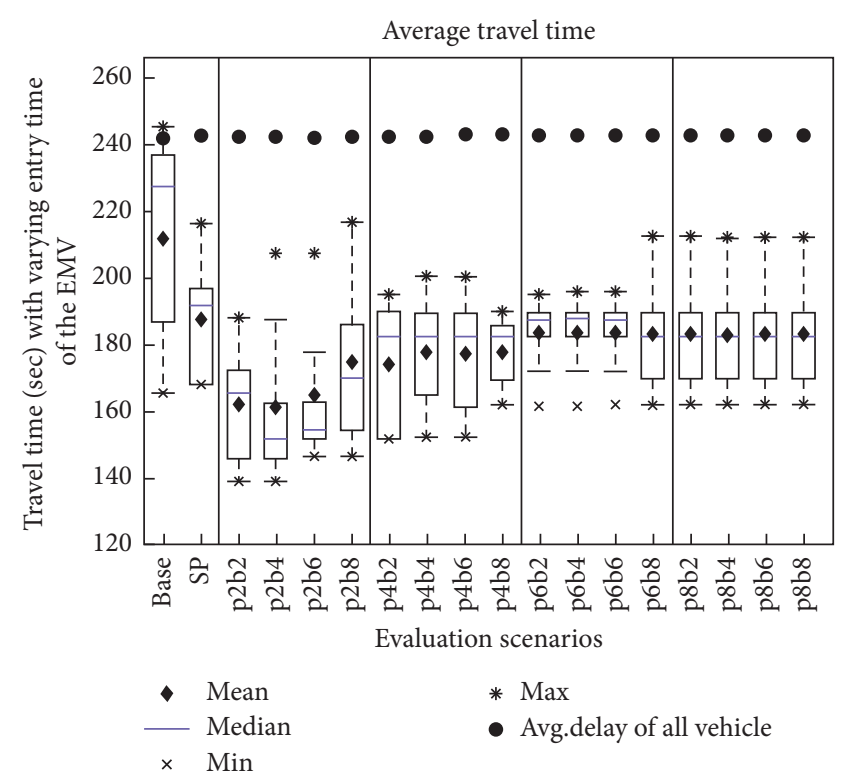

(b)

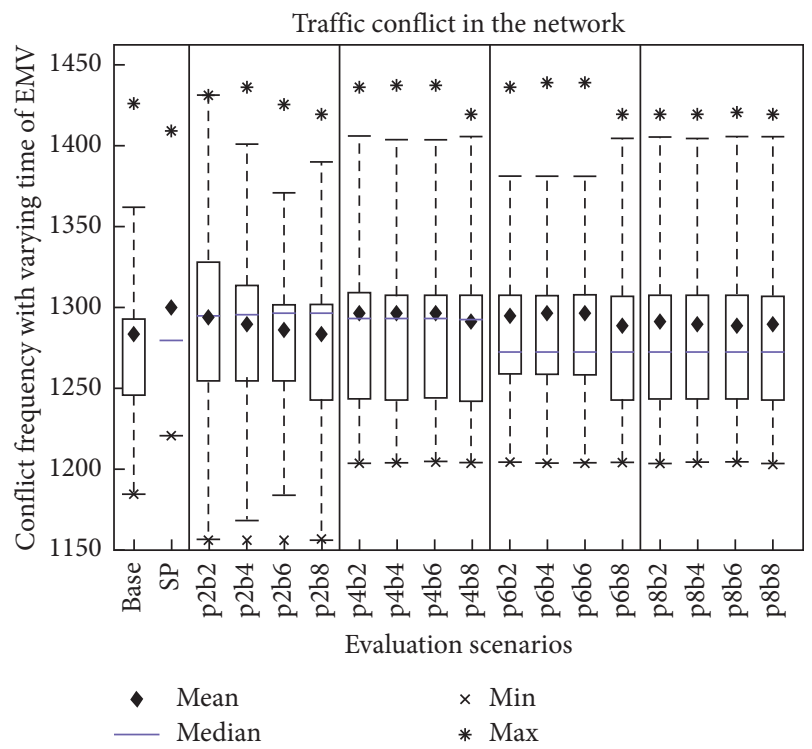

(d)

Figure 6: Safety and mobility assessment results. (a) Mobility measure, delay. (b) Mobility measure, travel time. (c) Safety measure, network-wide. (d) Safety measure, only EMV.

(v) The impacts on mobility and safety do not seem perfectly linear by the lane change parameter scenarios (16 parameter settings). This is because lane changes barely occurred when either the "benefit" parameter or the "possibility" parameter was set as $80 \%$, which is conservative. On the other hand, lane changes frequently occurred when either the "benefit" parameter or the "possibility" parameter was set as $20 \%$. This is the main reason behind the relationship between the parameter settings and the performance measures, but the other scenarios except the extreme settings appeared somewhat linear in the mobility and safety measures.
In order to find the reasonable level of the driving aggressiveness setting following the last finding in the above discussion, the optimum driving aggressiveness setting of EMV was captured in terms of mobility and safety. Figure 7 shows the performances of different EMV driving aggressiveness settings in two dimensions with the mobility and safety measures. Each data point represents the performance of each evaluation scenario including base, signal preemption, and 16 automated-control-integrated scenarios having different driving aggressiveness levels. In the most aggressive setting, the EMV can save approximately $20 \%$ of travel time compared to the p8b8 scenario (the most conservative setting), but $50 \%$ of traffic conflicts more occurred in the 


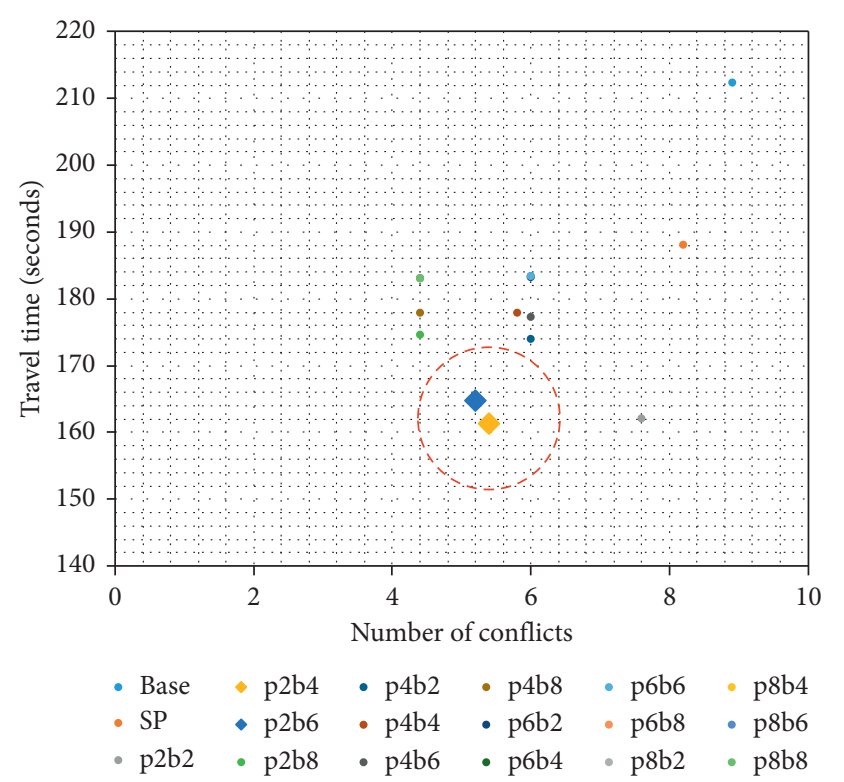

Figure 7: Multiobjective scatter plot.

p2b2 scenario (the most aggressive setting) compared to the most conservative setting. Although it could be still disputed what setting would be relevant for EMV, "p2b4" and "p2b6" settings (i.e., automated control in cooperation with signal preemption) appeared to be the most effective driving aggressiveness settings for EMV as the optimum driving aggressiveness setting in terms of both mobility and safety, which is the main objective of this study.

\section{Conclusions}

This study proposed an integrated approach of a signal preemption as the infrastructure-level traffic treatment and an automated driving control as the vehicle-level treatment in order to maximize the utilities of vehicle control including mobility and safety. Emergency vehicles (EMVs) such as ambulances and police vehicles were selected to implement this integrated vehicle control approach because the EMVs primarily need to reach their destinations fast and safe. Therefore, the integrated EMV control approach was developed based on the V2X wireless communications-based signal preemption strategy and the EIDM-based automated driving control, and the impacts of this automated EMV application were assessed with 16 different lane change aggressiveness levels (i.e., 16 different combinations of four lane change possibility criteria and four lane change benefit criteria) using a microscopic traffic simulation model. The measures of effectiveness included the driving maneuvers (lane change frequency, speed, and acceleration rate), the mobility measures (i.e., delay and travel time), and the safety measure (i.e., traffic conflicts).

The results showed that both the signal preemption and the automated driving control were beneficial to facilitate the EMV control in terms of mobility and safety, while they did not significantly provide negative impacts on adjacent normal vehicles. Importantly, the performance of the EMV was maximized when these two approaches were implemented at the same time. The results showed that the integrated approach was effective for the EMV to maintain its preset desired speed (i.e., $100 \mathrm{~km} / \mathrm{h}$ ) by providing a green passage at the signalized intersections and overtaking the lead vehicles driving in front of the EMV. In addition, the impact of automated vehicle control varies by different driving aggressiveness levels in terms of mobility and safety; aggressive settings having many lane changes performed well in terms of mobility, and the case was the opposite in terms of safety; and conservative settings having few lane changes were beneficial for safety, and the case was the opposite in terms of mobility. Therefore, these study results emphasize that the benefit of emergency vehicle control strategy can be maximized when the signal preemption and the reasonable levels of automated driving control settings operate in collaboration.

Nevertheless, this study should be enhanced with realistic behaviors of EMV and the other adjacent behaviors. Due to technical limitations on simulation modeling, this study was made with some assumptions that no special right-of-way (e.g., passing on red and driving on a contraflow lane) is allowed for EMV and adjacent vehicles do not take evasive maneuvers such as stopping and evasive road departure. Furthermore, the reasonable aggressiveness settings of automated driving control should be more investigated and validated through further studies. Besides, experimenting with additional scenarios including different traffic conditions and different penetration rates of other/ adjacent vehicles would help to validate the results and findings of this study.

However, this study showed benefits of the integrated approach of the automated driving technology at the individual vehicle level and the traffic signal preemption strategy at the traffic infrastructure level. In addition, this study showed potentials of automated driving controls: not only the safety aspect but also the mobility aspect. Therefore, this study will be a great reference and a starting point of discussion toward advanced automated driving technologies supporting not only safe but also fast mobility solution.

\section{Data Availability}

All experimental data (i.e., vehicle trajectories including $x / y /$ $z$, speed, and acceleration rate) used to support the findings of this study are available from the corresponding author upon request.

\section{Conflicts of Interest}

The authors declare that there are no conflicts of interest regarding the publication of this paper.

\section{Acknowledgments}

The authors thank researchers and colleagues of the Korea Transport Institute and the Chair of Traffic Engineering and Control at the Technical University of Munich for their sincere supports and advices. The first author especially thanks Mr. Liangyuan Tian (TED) for his sincere work in 
developing source codes for the automated vehicles simulation. It should be also noted that this work was supported by the Institute for Information \& Communications Technology Promotion (IITP) grant funded by the Korea Government (MSIT) (no. 2017-0-004000, development of 100 Mbps grade V2X and LTE communication system for cooperative driving).

\section{References}

[1] P. Koonce, Traffic Signal Timing Manual, Federal Highway Administration, Washington, DC, USA, 2008.

[2] X. Qin and A. M. Khan, "Control strategies of traffic signal timing transition for emergency vehicle preemption," Transportation Research Part C: Emerging Technologies, vol. 25, pp. 1-17, 2012.

[3] Federal Highway Administration, Traffic Signal Preemption for Emergency Vehicle a Cross-Cutting Study, US Federal Highway Administration, Washington, DC, USA, 2006.

[4] I. Yun, M. Best, and B. "Brian" Park, "Evaluation of transition methods of the 170E and 2070 ATC traffic controllers after emergency vehicle preemption," Journal of Transportation Engineering, vol. 134, no. 10, pp. 423-431, 2008.

[5] Y.-J. Lee, S. Dadvar, J. Hu, and B. B. Park, "Transit signal priority experiment in a connected vehicle technology environment," Journal of Transportation Engineering, Part A: Systems, vol. 143, no. 8, Article ID 05017005, 2017.

[6] Z. Li et al., "Intersection control optimization for automated vehicles using genetic algorithm," Journal of Transportation Engineering, Part A: Systems, vol. 144, no. 12, Article ID 04018074, 2018.

[7] Z. Lu, Q. Zhang, and X. Liu, "A formulation method of control protocol for intersection signal based on wireless communication technologies," in Proceedings of the ICTIS 2013: Improving Multimodal Transportation Systems-Information, Safety, and Integration, pp. 78-83, Wuhan, China, June 2013.

[8] K. Hou and J. Hu, "Vehicular networks communication analysis and speed guidance at signalized intersections," in Proceedings of the International Conference on Transportation and Development, Pittsburgh, PA, USA, July 2018.

[9] J. Levinson, J. Askeland, J. Becker et al., "Towards fully autonomous driving: systems and algorithms," in Proceedings of the Intelligent Vehicles Symposium (IV), IEEE, Dearborn, MI, USA, June 2011.

[10] R. Wiedemann, Simulation des Straßenverkehrsflusses, Schriftenreihe des Instituts für Verkehrswesen der Universität Karlsruhe, Karlsruhe, Germany, 1974.

[11] P. G. Gipps, "A behavioural car-following model for computer simulation," Transportation Research Part B: Methodological, vol. 15, no. 2, pp. 105-111, 1981.

[12] A. Kesting, M. Treiber, and D. Helbing, "Enhanced intelligent driver model to access the impact of driving strategies on traffic capacity," Philosophical Transactions of the Royal Society A: Mathematical, Physical and Engineering Sciences, vol. 368, no. 1928, pp. 4585-4605, 2010.

[13] PTV, PTV VISSIM 7 Introduction to the COM API, PTV, Islamabad, Pakistan, 2015.

[14] A. Kesting, M. Treiber, and D. Helbing, "General lanechanging model MOBIL for car-following models," Transportation Research Record: Journal of the Transportation Research Board, vol. 1999, no. 1, pp. 86-94, 2007.
[15] M. Treiber, A. Hennecke, and D. Helbing, "Congested traffic states in empirical observations and microscopic simulations," Physical Review E, vol. 62, no. 2, pp. 1805-1824, 2000.

[16] J. E. Naranjo, C. Gonzalez, R. Garcia, and T. de Pedro, "Lanechange fuzzy control in autonomous vehicles for the overtaking maneuver," IEEE Transactions on Intelligent Transportation Systems, vol. 9, no. 3, pp. 438-450, 2008.

[17] S. Ulbrich and M. Maurer, "Probabilistic online POMDP decision making for lane changes in fully automated driving," in Proceedings of the 16th International IEEE Conference on Intelligent Transportation Systems (ITSC 2013), IEEE, The Hague, The Netherlands, October 2013.

[18] E. A. Mueller, "Aspects of the history of traffic signals," IEEE Transactions on Vehicular Technology, vol. 19, no. 1, pp. 6-17, 1970.

[19] SAE, J2735 Dedicated Short Range Communications (DSRC) Message Set Dictionary, SAE, Warrendale, PA, USA, 2015.

[20] ETSI, TS 103301 Basic Set of Applications; Facilities Layer Protocols and Communication Requirements for Infrastructure Services, ETSI, Sophia Antipolis, France, 2016.

[21] K. Van Vliet and S. Turksma, ImFlow: Policy-Based Adaptive Urban Traffic Control, ITS Europe, Dublin, Ireland, 2013.

[22] K. Ahn, H. A. Rakha, K. Kang, and G. Vadakpat, "MMITSS impacts assessment: field testing and simulation results," in Proceedings of the Transportation Research Board 95th Annual Meeting Transportation Research Board, Washington, DC, USA, 2016.

[23] PTV VISSIM/VISWALK 10, PTV AG, 2017, http://vision-traffic. ptvgroup.com/fileadmin/files_ptvvision/Downloads_N/0_ General/2_Products/2_PTV_Vissim/Overview_PTVVissim10_ EN.pdf.

[24] G. Grigoropoulos, "Managing the growth and the Transport integration of BMW FIZ future," in Proceedings of the Transportation Systems Project Seminar, Technical University of Munich, Munich, Germany, June 2015.

[25] A. A. Rrecaj and K. M. Bombol, "Calibration and validation of the VISSIM parameters-state of the art," TEM JournalTechnology Education Management Informatics, vol. 4, pp. 255-269, 2015.

[26] H. D. Robertson, ITE Manual of Transportation Engineering Studies, Prentice Hall, Englewood Cliffs, NJ, USA, 1994.

[27] D. Gettman, Surrogate Safety Assessment Model and Validation: Final Report, Federal Highway Administration (FHWA), Washington, DC, USA, 2008. 